\title{
Treating anterior open bite
}

\section{Are orthodontic and orthopaedic treatments effective at correcting anterior open bite (dental, dento-alveolar and/ or skeletal) in children?}

\author{
Lentini-Oliveira D, Carvalho FR, Qingsong $Y$, et al. \\ Orthodontic and orthopaedic treatment for anterior open bite in \\ children. Cochrane Database Syst Rev 2007; issue 2
}

Data sources The Cochrane Central Register of Controlled Trials, Medline, Embase, LILACS (Latin American \& Caribbean Health Sciences Literature), Brazilian Bibliography of Odontology and SciELO. Ten Chinese journals were searched by hand and the bibliographies of papers were retrieved.

Study selection Randomised controlled trials (RCT) or quasi-RCT of orthodontic or orthopaedic treatments or both to correct anterior open bite in children were included.

Data extraction and synthesis Two review authors independently assessed the eligibility of all reports identified. Risk ratios (RR) and corresponding $95 \%$ confidence intervals $(\mathrm{Cl})$ were calculated for dichotomous data. The continuous data were expressed as described by the author.

Results Twenty-eight trials were potentially eligible, but only three RCT were included comparing treatments as follows: effects of Frankel's function regulator-4 (FR-4) with lip-seal training versus no treatment; repelling-magnet splints versus bite-blocks; and palatal crib associated with high-pull chincup versus no treatment. The study comparing repelling-magnet splints versus bite-blocks could not be analysed because the authors interrupted the treatment earlier than planned because of side-effects in four out of 10 patients. FR-4 associated with lip-seal training (RR, 0.02; 95\% Cl, 0.00-0.38) and removable palatal crib associated with high-pull chincup (RR, $0.23 ; 95 \% \mathrm{Cl}, 0.11-0.48$ ) were able to correct anterior open bite. No study described the randomisation process nor sample size calculation; there was not blinding in the cephalometric analysis; and the two studies also evaluated two interventions at the same time.

Conclusions There is weak evidence that the interventions FR-4 with lip-seal training and palatal crib associated with high-pull chincup are able to correct anterior open bite. Given that the trials included have potential bias, these results must be viewed with caution. Recommendations for clinical practice cannot be made based only on the results of these trials. More RCT are needed to elucidate the interventions for treating anterior open bite.

\section{Commentary}

Systematic reviews have advantages that benefit clinicians and academics alike but the evidence they provide is heavily dependent on the quality of available literature. One cannot make bricks without straw. A well-designed and performed systematic review does not always yield sound evidence on the effectiveness of a treatment modality. From this review, it is clear that evidence development on the orthodontic and orthopaedic treatment for anterior open bite in children is still in a premature phase at present.

Anterior open bite is regarded as one of the most difficult malocclusions to treat in orthodontics. Some clinicians do not even try to treat the problem because it is impossible to close the bite without deteriorating facial aesthetics, or is not warranted because of concerns over the long term stability of the closed bite.

Strictly speaking, the results showing effectiveness from the only two studies that were eligible could hardly be regarded as even 'weak' evidence. It simply results from two individual trials on two different treatment methods. Considering the possibility of publication bias (studies with significant results are more likely to get published than those with nonsignificant results) and the lack of statistical strength, either treatment modality could be recommended as 'effective'.

One comment on the conduct of the review is that in the searches, the non-English literature only included Brazilian and Chinese bibliographies. It is not clear whether trials in other languages have been checked or if no relevant publications were found.

It is known that Cochrane Reviews are based on the best available information about healthcare interventions. The fact that RCT represent a higher level of evidence does not mean that nonrandomised studies have no value. This review identified a large number of controlled trials evaluating anterior open bite treatment. They may collectively provide some informative guidance for clinicians in addition to the 'weak' evidence the authors gathered.

It could be a long wait before strong evidence appears on the treatment effectiveness for anterior open bite. Hopefully, the present dearth of evidence highlighted by this review will draw researchers' attention to establishing quality clinical trials in future. As a reflective exercise, we may revisit some remarks by Dr Eysenck, ${ }^{1}$ a psychologist, "If a medical treatment has an effect so recondite and obscure as to require meta-analysis to establish it, I would not be happy to have it used on me. It would seem better to improve treatment and the theory underlying the treatment".

\section{Yijin Ren \\ Department of Orthodontics, University Medical Centre Groningen, Groningen, The Netherlands \\ 1. Eysenck HJ. Systematic Reviews. Edited by Chalmers I, Altman DG. London: BMJ Publishing; 1995:p73. \\ Evidence-Based Dentistry (2007) 8, 5-6. doi:10.1038/sj.ebd.6400512}

